\title{
A Rare Form of Guillan Barre Syndrome: A Child Diagnosed with Anti- GD1a and Anti-GD1b Positive Pharyngeal-Cervical-Brachial Variant
}

\author{
Metin Uysalol ${ }^{1}$, Burak Tatlı1 , Nedret Uzel ${ }^{1}$, Agop Çıtak ${ }^{1}$, Erhan Aygün${ }^{1}$, Semra Kayaoğlu² \\ ${ }^{1}$ Department of Pediatrics, Istanbul University İstanbul Faculty of Medicine, Istanbul, Turkey \\ ${ }^{2}$ Department of Pediatrics, Şişli Etfal Training and Research Hospital, İstanbul, Turkey
}

\begin{abstract}
Background: Pharyngeal-cervical-brachial (PCB) variant is a rare form of Guillan-Barre Syndrome (GBS). Antibodies against other membrane proteins like GM1b and GD1a have been found only in a small number of patients with Guillan Barre syndrome variant.

Case Report: Here, we report a 5.5 year-old boy diagnosed early with positive GD1a and GD1b gangliosides of Guillan-Barre syndrome pharyngeal cervical-Brachial variant, who improved and recovered fully in a short period. This is in contrast to those whose recovery period prolongs in spite of early diagnosis and appropriate treatment and/or those who experience incomplete recovery.
\end{abstract}

Conclusion: In summary, diagnosis of PCB variant of GBS should be considered in infants with sudden onset bulbar symptoms and muscle weakness, and it should be kept in mind that early diagnosis and appropriate treatment can give successful outcomes.

Key Words: Guillain-Barré syndrome, pharyngeal-cervical-brachial variant, ganglioside antibodies, children, emergency department

Received: 10.12 .2012

Accepted: 01.06.2013

\section{Introduction}

Guillan-Barre Syndrome (GBS) is an acute post-infectious demyelinating polyneuropathy with an acute onset characterised by generally fast progressive muscle weakness and paraesthesia (1). GBS is diagnosed by clinical, laboratory and neurophysiological findings (2). Anti-ganglioside antibodies in patient serum support the diagnosis (3), while antibodies against other membrane proteins like GM1b and GD1a have been found in only a small number of patients (4). GBS has different clinical forms characterised by the different involvement of motor and sensory axons of peripheral nerves and the autonomic nervous system (5). The pharyngeal-cervicalbrachial (PCB) variant is a rare form of GBS with regional involvement. In adults, this variant shows itself especially with involvement and weakness of oropharyngeal, neck and upper extremity muscles (2). Although it was first stated that the leg muscle strength and reflexes were preserved in some patients, later, possible mild leg muscle weakness was also mentioned in some patients (2).

\section{Case Report}

A 5.5 year-old boy was admitted to the Pediatric Emergency Unit with complaints of weakness in his hands and arms, and difficulty in speech and walking which started a day ago. His family mentioned that he had a common cold which started a day ago with difficulty in coughing. His personal and family history revealed no specific information. His neurologic development was compatible with his age.
All his immunizations were on schedule. There was no recent immunization and no exposure to neurological agents. The first medical evaluation revealed that he was conscious and co-operative but restless. Pupils were isochoric and pupillary light reflexes were bilaterally positive. The patient had speech difficulty, dysarthria and rhinolalia. He had an unbalanced walk and had large steps without ataxia, as well as paralysis of both eyes when looking up and restrictive lateral sight. There was no ptosis of eyelids. His retching reflex was intact and tongue movements were normal. His face was meaningless and he had his mouth open. Deep tendon reflexes were bilaterally reduced at upper and lower extremities. The muscle strength was $4 / 5$ at neck flexor muscles, $3 / 5$ at distal and proximal muscles of the upper extremities and $4 / 5$ at distal and proximal muscles of lower extremities. There was no clonus or tremor. Plantar reflex response was flexion. The intestine and bladder sphincters were intact. Autonomic functions were normal. There was no sensory loss with touch and with needle prick. There was weak coughing. In the lungs, there were bilateral sonorous rales. Other system examinations were normal. Biochemical laboratory tests with ammonia, lactate and the haematological tests were normal. Retinal examination revealed no specific finding. No pathology was observed at the brain and brain stem by Magnetic Resonance Imaging. There was no microbial proliferation in cultures of blood, urine and stool. The results of routine serum virology studies and serum immunoglobulins were normal. The neostigmin test was negative. The serologic tests were performed for differential diagnosis and aetiology. The results were nega- 
tive for Herpes virus, Epstein-Barr virus, Cytomegalovirus, Rubella, Rubeola, Toxoplasmosis, Enteroviruses, Respiratory viruses, Lyme and Mycoplasma pneumoniae. There was no microbial proliferation in the culture for Salmonella, Shigella and Campylobacter jejuni. On the second day of hospital admission, the patient was taken to the intensive care unit due to difficulty in swallowing. Electromyography (EMG) performed the day after the onset of complaints revealed normal sensory amplitude and conductions. Right ulnar motor conduction evaluation revealed partial block. Other motor responses and conductions were normal. Right median and tibial $F$ responses were normal. Normal configuration motor unit potentials and sparseness were found in Needle EMG (Figures 1-3; Tables 1 and 2). Ophthalmoparesis (but no ptosis), bulbar involvement, upper extremity dominant side weakness and hypoactive deep tendon reflexes in this patient accompanied by acute neuropathy at neck, pharynx and upper extremity were defined as radiculopathy 'Guillan-Barre Syndrome Pharyngeal-Cervical-Branchial Variant'. Clinical and electrophysiological findings lead us to the diagnosis of GBS FSB variant. Assessment of the cerebrospinal fluid (CSF) taken by lumbar puncture, performed on the third day of onset of complaints, revealed no cells. In the cerebrospinal fluid, the protein level was $25 \mathrm{mg} / \mathrm{dL}$ and the glucose level was $96 \mathrm{mg} / \mathrm{dL}$. There was no microbial proliferation in cerebrospinal fluid cultures. Anti-ganglioside antibody tests were performed on the third day of onset of complaints. We examined IgG and IgM antibodies against Anti-GM1, Anti-GM2, Anti-GQ1b, Anti-GD1a and Anti-GD1b. AntiGM1 IgG 28.1\% (Negative: <30\%), Anti-GM2 IgG 29.81\% (Negative: <30\%), Anti-GQ1b IgG 28.32\% (Negative: $<30 \%$ ). Positive Anti-GD1a IgG (88.51\%; Normal: 30-50\%) and Anti-GD $1 \mathrm{~b}$ lgG (74.27\%; Normal: <30\%) ganglioside antibodies supported the diagnosis. Intravenous immunoglobulin (IVIG) was administered to the patient. There was fast clinical improvement following the therapy. There was mild weakness left only in an upper extremity on the fourth day of admission of the patient who was discharged from the hospital. Dysarthria was improving and he was being fed easier. Clinical findings improved almost 15 days after discharge from the hospital, while clinical and electrophysiological findings improved completely after a month.

Table 1. The Results of Analysis of Sensory Conduction

\begin{tabular}{|c|c|c|c|c|c|c|c|c|}
\hline Nerve/Sites & Rec. Site & $\begin{array}{c}\text { Peak Lat } \\
\text { ms }\end{array}$ & $\begin{array}{l}\text { Lat. } \\
\text { Ms }\end{array}$ & $\begin{array}{c}\text { Distance } \\
\mathbf{c m}\end{array}$ & $\begin{array}{c}\text { Velocity } \\
\mathrm{m} / \mathrm{s}\end{array}$ & $\begin{array}{l}\text { Amp. } \\
\text { 2-3 } \mu \mathrm{V}\end{array}$ & $\begin{array}{c}\text { Amp. } \\
1-2 \mu \mathrm{V}\end{array}$ & $\underset{\mu V}{\text { Ampl. }}$ \\
\hline \multicolumn{9}{|c|}{ R MEDIAN - Dig II Ortho } \\
\hline II.p_Wrist & Wrist & 2.20 & 1.75 & 9 & 51.4 & 32.2 & 19.8 & 32.2 \\
\hline \multicolumn{9}{|c|}{ R ULNAR - Digit V Ortho } \\
\hline V.p - Elbow & Elbow & 1.70 & 1.20 & 6 & 50.0 & 21.4 & 15.6 & 21.4 \\
\hline \multicolumn{9}{|c|}{ R SURAL - Lat Malleous } \\
\hline $\mathrm{Cr}$ & Lat Malleous & 2.15 & 1.60 & 7 & 43.8 & 19.2 & 14.9 & 19.2 \\
\hline
\end{tabular}

Table 2. The Result of Analysis of Motor Conduction

\begin{tabular}{|c|c|c|c|c|c|c|c|c|}
\hline Nerve/Sites & Latency ms & $\begin{array}{c}\text { Ampl } \\
\mathbf{m V}\end{array}$ & $\begin{array}{c}\text { Distance } \\
\text { cm }\end{array}$ & $\begin{array}{c}\text { Velocity } \\
\mathrm{m} / \mathrm{s}\end{array}$ & $\begin{array}{l}\text { Dur. } \\
\text { ms }\end{array}$ & $\begin{array}{c}\text { Area } \\
\text { mV } \\
\text { ms }\end{array}$ & $\begin{array}{c}\text { Amp. } \\
2-4 \\
m V\end{array}$ & $\begin{array}{c}\text { D Lat } \\
\text { ms }\end{array}$ \\
\hline \multicolumn{9}{|c|}{ R MEDIAN - APB } \\
\hline Wrist & 2.70 & 5.4 & & & 4.35 & 13.3 & 7.3 & 2.70 \\
\hline Elbow & 5.30 & 5.2 & 15 & 57.7 & 4.40 & 12.9 & 7.1 & 2.60 \\
\hline \multicolumn{9}{|c|}{ R ULNAR - ADM } \\
\hline Wrist & 1.80 & 4.3 & & & 5.20 & 12.6 & 6.4 & 1.80 \\
\hline Below Elbow & 5.05 & 1.0 & 18 & 55.4 & 5.40 & 2.5 & 1.5 & 3.25 \\
\hline \multicolumn{9}{|c|}{ R COMM PERONEAL - EDB } \\
\hline Wrist & 2.60 & 4.5 & & & 5.30 & 13.1 & 7.7 & 2.60 \\
\hline Fibular head & 8.10 & 3.7 & 23 & 41.8 & 5.40 & 11.6 & 6.1 & 5.50 \\
\hline \multicolumn{9}{|c|}{ R TIBIAL (KNEE) - AH } \\
\hline Wrist & 3.05 & 6.4 & & & 5.80 & 18.1 & 11.3 & 3.05 \\
\hline Popliteal & 9.05 & 4.6 & 22.5 & 37.5 & 6.70 & 16.0 & 8.0 & 6.00 \\
\hline
\end{tabular}




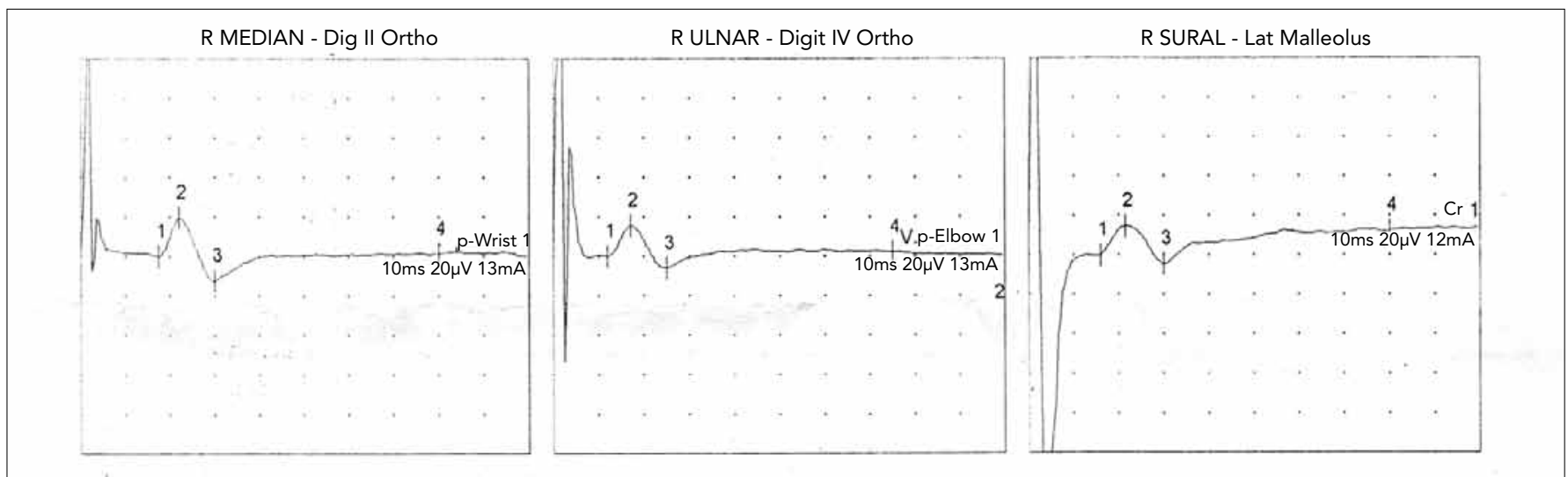

Figure 1. Sensory Conduction Analysis.

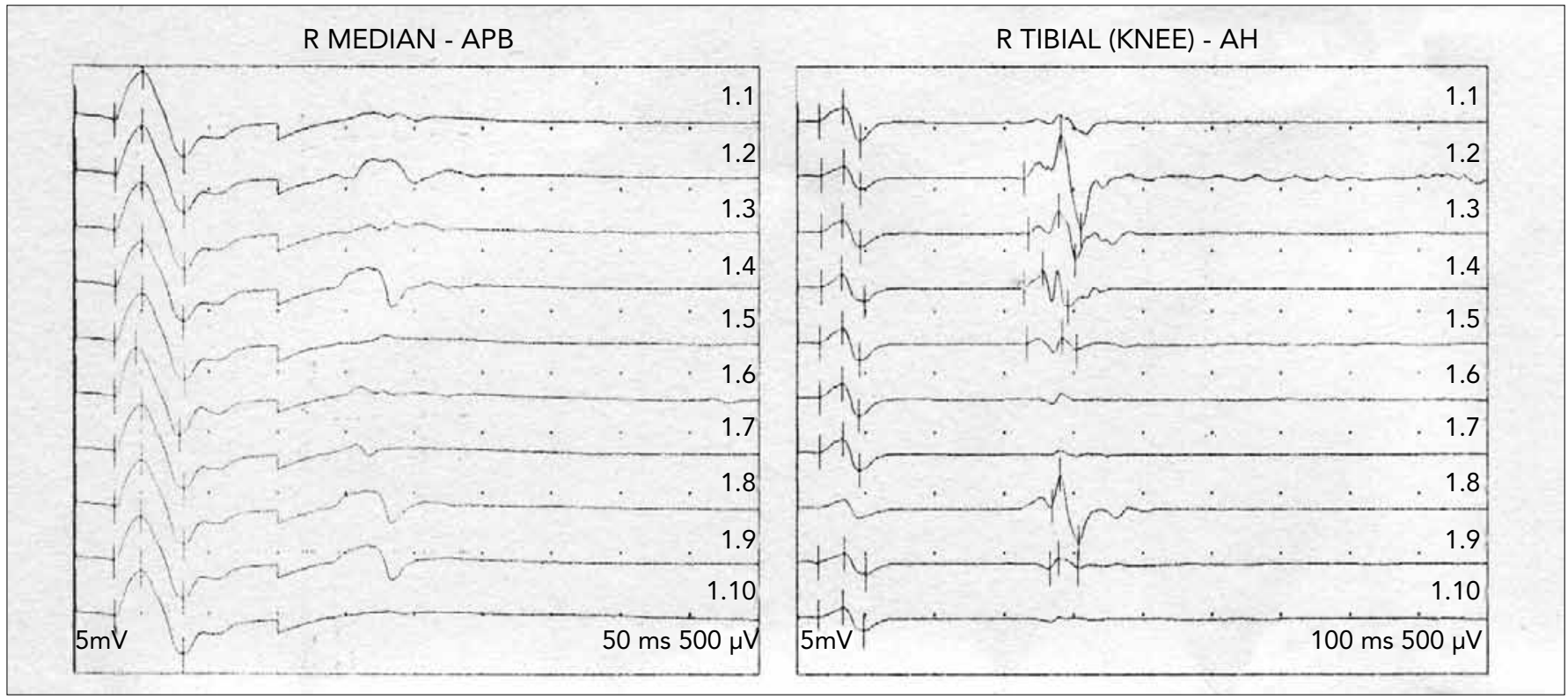

Figure 2. F wave. APB: Abductor pollicis brevis; AH: Abductor hallucis

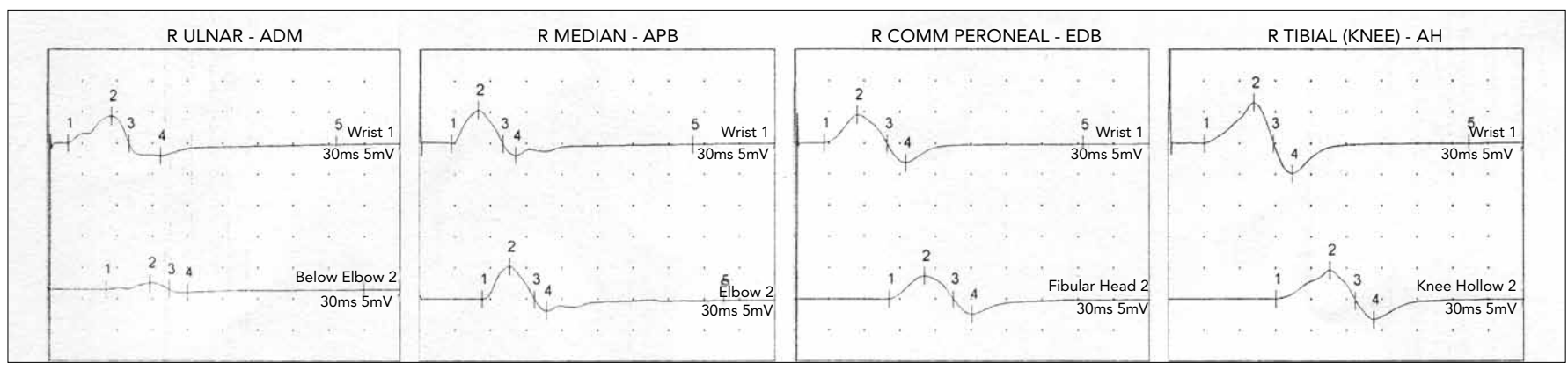

Figure 3. Motor Conduction Analysis. APB: Abductor pollicis brevis; ADM: Abductor digit minimi; EDB: Extensor digitorum brevis; AH: Abductor hallucis

\section{Discussion}

GBS is typically characterized by progressive symmetric paralysis, loss of deep tendon reflex, various degree of sensory loss or paraesthesia, albumin cytological dissociation and demyelination at peripheral nerves detected at electrophysiological nervous system conduction analysis and axonal degeneration. Marked extremity weakness along with facial nerve paralysis and ophthalmoplegia have been reported in $45 \%$ of affected children. In $15 \%$ of cases, proximal muscle weakness might be more prominent (6). There are different variants of GBS manifesting themselves with atypical clinical and electrophysiological findings. Acute inflammatory demyelinating polyneuropathy, acute motor axonal neuropathy, 
acute motor and sensory axonal neuropathy, acute sensory neuropathy, Miller Fisher syndrome, cranial polyneuritis, acute pandisotonomia and PCB variant are the forms that are seen in the spectrum (5). The prominent finding in these variants is cranial nerve involvement. Recently, the detection of clinical and pathological overlapping signs of different subtypes of GBS like Fisher Syndrome, PCB variant and Bickerstaff brain stem encephalitis lead us to believe that this situation probably represents forms of consistent processes versus different variants $(7,8)$.

Ropper defined 3 cases of PCB variant of GBS with weakness of the pharynx, neck, shoulder and arm, and difficulty in swallowing but with normal sensory response. In these patients, extremities being intact was thought to be diagnostic (2). Later, in some patients, mild muscle weakness at lower extremities was defined $(2,4,6,9)$. In our case, there was weakness of the oropharynx, neck, shoulder and arm muscles, and difficulty in swallowing. Sensory response was normal. There was marked weakness of muscle at upper extremities along with mild muscle weakness at lower extremities.

PCB variant of GBS in children is rare. A sixteen monthold child with gastroenteritis that started 2 weeks before the onset of symptoms was reported as a Pharyngeal-CervicalBrachial Variant of Guillan Barre Syndrome (10). Another report revealed a 12 year-old patient who was first diagnosed as epiglottis when admitted with acute respiratory distress and, while bulbar involvement was shown lately, the current diagnosis had delayed (11). Acute demyelinating PCB variant in 3 and 10 year-old children was shown in a study; in the latter, there was previous history of meningococcal vaccination (12). An another study showed that a 15 year-old girl had developed bulbar palsy and mainly upper extremity weakness two weeks after cytomegalovirus infection (13). It might be difficult to diagnose and diagnosis could be delayed in GBS variants with atypical findings. Our patient was lucky to recover in a short time with quick treatment, as rapid diagnosis was made by clinical and laboratory findings.

In CSF analysis, findings can be normal or with mild increased protein in the early period (4). In our case, there was no specific finding in CSF in the early period. In nerve conduction analysis in PCB variants of GBS, generally acute, low amplitude compounded muscle action potentials along with motor axonal damage and intact sensory reactions are observed. Conduction speed is typically normal. $\mathrm{F}$ and $\mathrm{H}$ waves and winking reactions are generally abnormal (12). However, in some cases, normal electro diagnostic findings or primary demyelination process findings have been reported (2). While evaluating paediatric cases, 3 demyelinating involvements and 3 axonal involvements were reported as different from adult cases (9-11). The difference in adult and child cases though is interesting, and is based on restricted number of observations. In our case, electrophysiological evaluation was compatible with PCB variant. Normal findings were found at one month follow-up.

In GBS, there are often upper respiratory and gastrointestinal infections before the disease (2). In our case, serological tests were negative in spite of respiratory infection at medical history and examination findings. In GBS and its variants, anti-ganglioside antibodies might be detected in patients' serum. Anti-ganglioside antibodies are thought to be useful while supporting the diagnosis (3). Acute motor-sensory axonal neuropathy (AMSAN): GM1, GM1b, GD1a; acute motor axonal neuropathy (AMAN): GM1, GM1a, GD1a, GalNacGD1a; Miller-Fisher syndrome: GQ1b, GT1a GQ1b; MillerFisher syndrome/GBS overlap syndrome: GQ1b, GM1, GM1a, GD1a, GalNac-GD1a; acute inflammatory demyelinating polyradiculoneuropathy (AIDP): antibodies unknown; Miller-Fisher syndrome/GBS overlap syndrome: GQ1, GM1, GM1a, GD1a, GalNac-GD1a. On the contrary, IgG antibodies to GM1b, GD1a, and GT1a have been reported in several patients with PCB. $(1,3,4)$. Anti-ganglioside GQ1b antibodies were shown in Fisher syndrome and polyneuritis cranialis (14). A case reported a girl with increased isolated GT1a anti-lgG antibodies and $P C B$ variant of GBS developed after cytomegalovirus infection (13). Another report showed that GT1a-sepecific IgG antibodies are positive for Clostridium jejuni in all GBS variants (15). Antibodies against other membrane glycolipids like GD1a and GM1b have been found in small number of variants of GBS (4). In the case of a patient with PCB variant of GBS, anti-GD1a was reported to show lower activity compared to anti-GT1a $\lg G$ (6). It was indicated in another study that the lower cranial nerves showed a higher rate of anti-GD1a antibody (14). Thus, it was thought that anti-GD1a in some patients with PCB of GBS caused bulbar palsy. In our case, rare Anti-GD1a lgG and Anti-GD1b lgG antibodies have been found to be positive. The positivity of Anti-GD1b lgG has not yet been reported. The positivity of Anti-Gd1b was commonly determined in case of GBS with ataxia and in some Miller-Fisher Syndrome cases (3). In this case, no ataxia was observed. Most Anti-lgGD1b antibodies are found to be associated with sensory neuropathy (3). However, our study did not show this finding. A case of an adult patient with positivity of GBS on axonal PCB variant has also been reported (4). This situation shows that GBS is still an example of a complex structure involving various signs and symptoms.

Although the prognosis information on PCB variant of GBS cases is limited in children, a prolonged recovery phase and incomplete improvements have been reported in adults $(2,6,10)$. Electro diagnostic indicators showing serious axonal damage were thought to play no leading role in recovery in children with GBS (1). Our case had mild bulbar signs, although muscle involvement distribution was compatible with PCB. The patient had almost recovered on the third day, the clinical signs had regressed on the fifteenth day, clinical and electrophysiological signs had completely improved. When compared with other cases, our patient showed complete improvement both on clinical and laboratory results after a month of an impressive course of treatment. In adult cases, prolonged incomplete improvements were reported. A weak and incomplete improvement was observed in spite of treatment in two cases of PCB variant of GBS which had Anti-GD1a IgG positivity and Anti-GD1b positivity, as in our case (4). Although the complete recovery of a 10 year-old patient with diagnosis of PCB variant of GBS took 3 months, the full recovery of a 3 year-old patient even after 3 months did not occur (12). The cases with bulbar paralysis and ophthalmoparaesis at the 
onset of the disease took longer (i.e. 5 months) to recover versus others (15). In this rare case, early and appropriate treatment was performed as a result of early diagnosis leading to very good results.

The aim in GBS treatment is to stop the autoimmune process causing destruction of the peripheral nerve myelin sheath by early immunomodulator therapy. The high dose of IVIG administration is believed to reduce auto-antibodies by binding the complement and repressing the production of $\mathrm{B}$ cell-mediated antibodies. In Childhood GBS, IVIG is given at days 2-5, and the clinical response becomes pronounced at 3-7 days (4). Likewise, as the clinical state of our patient first progressed, it quickly restored after IVIG administration.

In conclusion, the diagnosis of GBS and variants should be considered in babies and infants admitted to clinic with the sudden onset of bulbar palsy syndromes and/or with muscle weakness. Further information and the creation of more awareness about PCB of GBS variant would help with early diagnosis, timely and appropriate administration of treatment.

Ethics Committee Approval: N/A.

Informed Consent: Written informed consent was obtained from the parent of the patient.

Peer-review: Externally peer-reviewed.

Author contributions: Concept - M.U., N.U.; Design - M.U., N.U., B.T., A.Ç.; Supervision - M.U., N.U.; Resource - M.U., N.U.; Materials - M.U., E.A., S.K.; Data Collection\&/or Processing - M.U., A.Ç., B.T., E.A., S.K.; Analysis\&/or Interpretation - M.U., S.K.; Literature Search - M.U., E.A.; Writing - M.U., N.U.; Critical Reviews - M.U, N.U.

Conflict of Interest: No conflict of interest was declared by the authors.

Financial Disclosure: No financial disclosure was declared by the authors.

\section{References}

1. Doom PA, Ruts L, CJacops B. Clinical features, pathogen-esis, and treatment of Guillain-Barré syndrome. Lancet Neurol 2008;7:939-50. [CrossRef]
2. Ropper AH. Unusual clinical variants and signs in Guillain-Barré syndrome. Neurological and neurosurgical intensive care. Lippincott Williams \& Wilkins, 2004:278-97.

3. Willison HJ, Yuki N. Peripheral neuropathies and anti-glycolipid antibodies. Brain 2002;125: 2591-625. [CrossRef]

4. Arai M, Susuki K, Koga M. Axonal pharyngeal-cervical brachial variant of Guillain-Barré syndrome without anti-GT1a lgG antibody. Muscle Nerve 2003;28:246-50. [CrossRef]

5. Winer JB. Gullian Barre syndrome: clinical variant and their pathogenesis. J Neuroimmunol 2011;231:70-2. [CrossRef]

6. Mizoguchi K, Hase A, Obi T, Matsuoka H, Takamatsu M, Nishimura $Y$, et al. Two species of anti ganglioside antibodies in a patient with a pharyngeal-cervical-brachial variant of Guillain-Barre syndrome. J Neurol Neurosurg Psychiatry 1994;57:1121-3. [CrossRef]

7. Delanoe C, Sebire G, Landrieu P, Huault G, Metral S. Acute inflammatory demyelinating polyradiculopathy in children: Clinical and electrodiagnostic studies. Ann Neurol 1998;44:350-6. [CrossRef]

8. Nagashima T, Koga M, Odaka M, Hirata K, Yuki N. Continuous spectrum of pharyngeal-cervical-brachial variant of GuillainBarre syndrome. Arch Neurol 2007;64:1519-23. [CrossRef]

9. Levin KHM. Variants and mimics of Guillain-Barré syndrome. Neurologist 2004;10:61-74. [CrossRef]

10. Hergüner $M O$, Tepe $T$, Altunbaşak $S$, Baytok V. A rare form of Guillain-Barr ésyndrome: pharyngeal-cervical-brachialvariant. Turk J Pediatr 2008;50:91-3.

11. McLennan SC, Fahey MC, Lawson JA. Pharyngeal-cervical-brachial variant Guillain-Barré syndrome in a child. J Child Neurol 2004;19:626-7. [CrossRef]

12. Mogale KD, Antony JH, Ryan MM. The pharyngeal-cervicalbrachial form of Guillain-Barr é syndrome in childhood. Pediatr Neurol 2005;33:285-8. [CrossRef]

13. Murakami N, Tomita Y, Koga M, Takahashi E, Katada Y, Sakuta R, et al. An adolescent with pharyngeal-cervical-brachial variant of Guillain- Barré syndrome after cytomegalovirus infection. Brain Dev 2006;28:269-71. [CrossRef]

14. Chiba A, Kusunoki S, Obata H, Machinami R, Kanazawa I. Ganglioside composition of the human cranial nerves, with special reference to pathophysiology of Miller-Fisher syndrome. Brain Res 1997;745:32-6. [CrossRef]

15. Koga M, Yuki N, Hirata K. Antiganglioside antibody in patients with Guillain-Barre acute; syndrome who show bulbar palsy as an initial symptom. J Neurol Nearosurg Psychiatry 1999;66:513-6. [CrossRef] 